\title{
RELACIÓN ENTRE EL CLIMA LABORAL Y LA SATISFACCIÓN DEL CLIENTE EXTERNO DE DARUCHI SOLUTIONS S.A.C. COMO PARTE DE SU ESTRATEGIA DE INTERNACIONALIZACIÓN
}

\author{
RELATION BETWEEN WORK CLIMATE AND \\ EXTERNAL CLIENT SATISFACTION OF \\ DARUCHI SOLUTIONS S.A.C. AS PART OF ITS \\ INTERNATIONALIZATION STRATEGY
}

Recepción: 2018-12-26 - Aceptación: 2019-09-26

\section{Carmen Christina Guerra Huayllasco ${ }^{1 ;}$ a}

(iD) ORCID iD 0000-0001-5157-4950

\section{Luisa Adriana Ávila Bolívar ${ }^{1 ;}$ b}

(iD) ORCID iD 0000-0002-4746-5644

1 Universidad Ricardo Palma. Facultad de Ciencias Económicas y Empresariales. Escuela Profesional de Administración de Negocios Globales.

a Licenciada en Administración de Negocios Globales.

b Magister en Administración de Empresas.

\section{RESUMEN}

Objetivo: Determinar la relación entre el clima laboral con la satisfacción del cliente externo de Daruchi Solutions S.A.C. como parte de su estrategia de internacionalización. El tipo de investigación es correlacional. Métodos: Deductivo-explicativo. Entre los resultados más importantes se destacan que cuatro de las nueve dimensiones del Clima Laboral tienen relación con la satisfacción del cliente de la empresa Daruchi Solutions S.A.C. Es decir, que la apreciación de los trabajadores con respecto a las dimensiones: estructura, calor, apoyo e identidad, tienen relación con la la satisfacción del cliente. Conclusiones: El clima laboral en Daruchi Solutions S.A.C. en general no tiene relación con la satisfacción del cliente externo de Daruchi Solutions S.A.C., debido a que no todas las dimensiones del clima laboral tienen relación con la satisfacción del cliente externo de Daruchi Solutions S.A.C. Por otro lado, se halló que el clima laboral de la empresa es favorable.

Palabras clave: Clima laboral; Satisfacción del cliente; Importadora; Internacionalización.

\section{ABSTRACT}

The objective of the research was to determine the relationship among work climate and external client's satisfaction of Daruchi Solutions S.A.C. as part of its internationalization strategy. The type of research was correlational. The deductive-explanatory method was used. Among the most important results, stand out four from nine dimensions of Work Climate which have relation with client's satisfaction of Daruchi Solutions S.A.C. In other words, worker's assessment regarding the dimensions: structure, warmness, support, and identity have relation with client's satisfaction. However, it was concluded that work climate at Daruchi Solutions S.A.C. in general does not have relation with external client's satisfaction of Daruchi Solutions S.A.C; on account of not all work climate's dimensions have relation with client satisfaction of Daruchi Solutions S.A.C. On the other side, it was found that the work climate inside the company is favorable.

Key words: Work climate; Client satisfaction; Importer; Internationalization. 


\section{INTRODUCCIÓN}

Novoa y Regalado (2017) explicaron que su principal objetivo fue estudiar la relación existente entre el clima laboral y la satisfacción de los clientes en la empresa Oltursa. Utilizaron un diseño descriptivo transeccional. Su muestra estuvo conformada por 340 clientes y realizaron el análisis estadístico multivariado. Entre sus conclusiones resalta el hallazgo de la existencia de una relación significativa entre el clima laboral y la satisfacción de los clientes.

En la investigación de Montoya (2016) se observó que su objetivo fue estudiar y establecer la relación entre el clima organizacional y la evaluación del desempeño. Ha utilizado el método transversal correlacional. Dentro de sus principales conclusiones se pudo observar que existe una relación directa entre el clima organizacional y el desempeño laboral o la manera en que ejecutan sus tareas, en los trabajadores de la empresa PTS Perú.

Por otro lado, el objetivo de Sierra (2015) fue únicamente investigar las condiciones del clima laboral dentro del Hospital Regional de Cobán, el cual permitió establecer cuáles fueron los aspectos influyentes en la comunicación; además se propuso una guía metodológica, la cual permitió fortalecer al clima laboral del Hospital, específicamente dentro del área administrativa del Hospital. Ha utilizado el método descriptivo. Su principal conclusión fue que el clima laboral del Hospital Regional de Cobán, específicamente del área administrativa se encuentra favorable, debido a los factores positivos encontrados. Sin embargo, la alineación a la calidad y la remuneración son un tanto deficiente.

También se halló dentro de la investigación realizada por Echegaray (2014) que su principal objetivo fue determinar cómo se relacionan el clima organizacional con la satisfacción del cliente de la CMAC TACNA. El diseño utilizado por el investigador es descriptivo - correlacional. Se tuvo como su principal conclusión que el clima organizacional influye de manera significativa en la satisfacción de la empresa mencionada anteriormente.

De la misma manera, Pelaes (2010) establece como su principal objetivo el determinar la existencia de una relación directa entre el clima organizacional y la satisfacción del cliente de la empresa Telefónica, de modo que el incremento del nivel del clima organizacional se viera reflejado en el aumento de la satisfacción de los clientes. El diseño descriptivo - correlacional fue utilizado para esta investigación. Entre sus principales conclusiones se tuvo que, efectivamente, existe una relación entre el clima organizacional y la satisfacción de los clientes de la empresa.

La investigación de Cortés (2009) trazó como objetivo principal diagnosticar las dimensiones del clima laboral u organizacional en el Hospital Dr. Luis F. Nachón, además permitir a la organización estar al tanto del grado de motivación de los empleados del hospital, desde la perspectiva de los mismos. La investigadora ha utilizado el método descriptivo transversal y observacional. Dentro de sus principales conclusiones se pudo observar que el clima organizacional general en el hospital mencionado líneas arriba no es del todo satisfactorio, también se concluyó que existe preocupación por parte de los directivos para que el personal logre la comprensión idónea del trabajo; que la repartición del trabajo es organizada y que existe disposición de efectuar los objetivos establecidos del departamento.

Finalmente, Echezuría y Rivas (2001) plantearon el objetivo de determinar el clima organizacional de los organismos públicos y de la empresa privada en Carcas - Venezuela en el año 2001, el cual permitió comparar los resultados obtenidos en la medición del clima organizacional de los organismos públicos con los de las empresas privadas. Las investigadoras utilizaron el método descriptivo-explicativo. Su conclusión principal expresó que existen diferencias notables en las apreciaciones de las dimensiones. La mayor brecha se encontró en las dimensiones Conflicto, Recompensa, Estándares de Desempeño, Estructura y Responsabilidad.

\section{Diagnóstico de la situación problemática}

Se han presentado deficiencias internas, que repercuten en la operatividad de la empresa en general, que se detallan a continuación:

a) Los manuales de organización y funciones se encuentran desactualizados;

b) Inexistencia de comunicación de estrategias en forma clara;

c) Carencia de trabajo en equipo para dar a conocer a los empleados en detalle el propósito de determinados trabajos que se les encomiendan.

Al analizar la situación, se determinaron los siguientes supuestos de las posibles causas:

a) Un clima laboral con ciertas deficiencias que no permiten una óptima productividad.

b) Endeble trabajo en equipo para dar a conocer a los empleados en detalle el propósito de determinados trabajos que se les encomiendan.

Por otro lado, la gerencia de Daruchi Solutions, tiene la intención de internacionalización, es decir, de expandir la empresa en mercados de la región, si bien es cierto dentro del plan estratégico no está plasmado un plan específico sobre la internacionalización, sí que es verdad que ser una empresa importadora está dentro de la visión, el cual es el paso inicial para empezar con la internacionalización. 
Pregunta de investigación:

¿Cuál es la relación entre el clima laboral yla satisfacción del cliente externo de Daruchi Solutions S.A.C. como parte de su estrategia de internacionalización?

Hipótesis:

El clima laboral en Daruchi Solutions S.A.C. tiene relación con la satisfacción del cliente externo de Daruchì Solutions S.A.C.

\section{Objetivo:}

Determinar la relación entre el clima laboral y la satisfacción del cliente externo de Daruchi Solutions S.A.C. como parte de su estrategia de internacionalización.

Debido a que la empresa cuenta con la intención de internacionalización, empezando por ser una empresa importadora de marcas transnacionales de tecnología informática tales como Dell, HP, Intel, Lenovo, Microsoft, Cisco, APC, Adobe, Mcafee, entre otras; la importancia de la presente investigación radicó en que, al medir el clima laboral y la satisfacción del cliente externo se obtuvo información sobre si existe una relación entre el clima laboral y la satisfacción del cliente externo.

\section{MATERIAL Y MÉTODOS}

\section{Población y muestra}

La población de la variable independiente (Clima Laboral) está comprendida por la totalidad de los trabajadores (10) del área más crítica de Daruchi Solutions S.A.C. Así mismo, la población de la variable dependiente (Satisfacción del Cliente externo) está comprendida por sus clientes más destacados (10) del área de soporte de tecnologías de Daruchi Solutions S.A.C.

La muestra para la variable Clima Laboral es de 10 trabajadores, es decir la totalidad de la población, por lo tanto, es una muestra censal ya que es la cantidad que representa a la población en su totalidad. Lo mismo sucede para la variable Satisfacción del Cliente Externo, la muestra es de 10 clientes, los cuales representan la totalidad de la población, por tanto, es una muestra censal.

\section{Recolección y procesamiento de datos}

Recolección de datos:

El instrumento de recogida de datos fue el cuestionario.

Para medir el clima laboral, el cuestionario de Litwin y Stringer, quienes validaron dicho instrumento, fue aplicado en su forma original a los 10 trabajadores. Constaba de 50 preguntas divididas en 9 dimensiones con una escala de Likert del 1 al 4.
Para medir la satisfacción del cliente, el cuestionario que se aplicó a los 10 clientes fue el modelo SERVQUAL, el cual fue elaborado y validado por Zeithaml, Parasuraman y Berry (como se citó en Matsumoto, 2014), conformado por 22 preguntas divididas en 5 dimensiones con una escala de Likert del 1 al 7. De acuerdo con Parasuraman, Zeithaml y Berry (1988):

"SERVQUAL is a concise multiple-item scale..., it provides a basic skeleton through its expectations/perceptions format encompassing statements for each of the five service-quiality dimensions...When neccesary can be adapted or supplemented to fit ... the specific research needs of a particular organization". [SERQUAL es una escala concisa de elementos múltiples..., provee un esqueleto básico a través de su formato de expectativas/percepciones incluyendo afirmaciones para cada una de las cinco dimensiones de calidad de servicio...Cuando sea necesario puede ser adaptada o complementada para calzar a las necesidades específicas de investigación de una organización en particular]. (p.30-31)

Tomando en cuenta lo mencionado por los autores líneas arriba, se precedió a utilizar únicamente el cuestionario en sí, de manera que no se realizó el análisis profundo que propone la metodología de SERVQUAL debido a que la presente investigación ha sido enfocada principalmente al análisis del clima laboral de la empresa.

\section{Procesamiento de datos:}

Una vez culminadas las encuestas con escalas de Likert, se aplicaron criterios estadísticos para la tabulación de cuadros, utilizando el software SPSS versión 24. Para la presente investigación se utilizó las siguientes técnicas de procesamiento: tabulación y elaboración de tablas de distribución de frecuencia. Además, se realizaron los análisis utilizando la prueba Chi-cuadrado, para la luego realizar la interpretación.

\section{RESULTADOS}

Los resultados que se muestran a continuación están estructurados de la siguiente manera:

a. Resumen del cuestionario para medir el clima laboral: La tabla e interpretación mostradas en esta sección permiten conocer el estado del clima laboral dentro de la empresa, que en este caso el clima laboral es favorable.

b. Resumen del cuestionario para medir la satisfacción del cliente: La tabla e interpretación mostradas en esta sección permiten conocer el estado de la satisfacción del cliente, en este caso hay un alto porcentaje de satisfacción por parte del cliente.

c. Resultados de la prueba de hipótesis: En esta sección se encuentra el resultado final, el cual surge al cruzar la información de las tablas mencionadas 
líneas arriba. En este caso, se rechaza la hipótesis, por lo tanto, no hay relación entre el clima laboral y la satisfacción del cliente.

\section{Resumen del cuestionario para medir clima laboral}

A continuación, en la Tabla 1 se muestra el resumen de las respuestas del cuestionario, de las 9 dimensiones del Clima Laboral, aplicado a los trabajadores para medir el estado del clima laboral dentro de la empresa. De acuerdo con los resultados que muestra dicha tabla, el clima laboral dentro de la empresa Daruchi Solutions S.A.C. en términos generales es favorable, debido a que el $91 \%$ de los trabajadores se encuentran entre parcialmente y altamente de acuerdo con todas las dimensiones pertenecientes al clima laboral, estas son: estructura, responsabilidad, recompensa, riesgo, calor, apoyo, estándares de desempeño e identidad. Sin embargo, aún hay dimensiones que deben ser reforzadas o mejoradas, para así tener a la mayoría de los trabajadores altamente de acuerdo con el clima laboral de la empresa; ya que actualmente solo el $42 \%$ se encuentra altamente de acuerdo con esta.

Tabla 1

Conjunto de condiciones sociales y psicológicas que caracterizan a la empresa, y que repercuten de manera directa en el desempeño de los empleados.

\begin{tabular}{lll}
\hline Respuesta & $\begin{array}{l}\text { Frecuencias de } \\
\text { respuestas }\end{array}$ & Porcentajes \% \\
\hline $\begin{array}{l}\text { Altamente en } \\
\text { desacuerdo }\end{array}$ & 1 & 1,0 \\
$\begin{array}{l}\text { Parcialmente en } \\
\text { desacuerdo }\end{array}$ & 7 & 8,0 \\
$\begin{array}{l}\text { Parcialmente de } \\
\text { acuerdo }\end{array}$ & 44 & 49,0 \\
$\begin{array}{l}\text { Altamente de } \\
\text { acuerdo }\end{array}$ & 38 & 42,0 \\
Total & 90 & 100,0 \\
\hline
\end{tabular}

Fuente: Datos de la propia investigación.

Resumen del cuestionario para medir la satisfacción del cliente

De la misma manera, en la Tabla 2 se muestra el resumen de las respuestas del cuestionario para medir la satisfacción de los clientes de Daruchi Solutions S.A.C. Dicha tabla muestra que el $70 \%$ de los clientes de la empresa se encuentran altamente de acuerdo con los servicios recibidos. Esto significa que la empresa está cumpliendo con las expectativas de la mayoría de sus clientes.

\section{Prueba de Hipotesis}

Hipótesis planteada: El clima laboral en Daruchi Solutions S.A.C. tiene relación con la satisfacción del cliente externo de Daruchi Solutions S.A.C.
Tabla 2

Diferencia de medias: grupo A y grupo $B$

\begin{tabular}{lll}
\hline Respuesta & $\begin{array}{l}\text { Frecuencias de } \\
\text { respuestas }\end{array}$ & Porcentajes \% \\
\hline $\begin{array}{l}\text { Altamente en } \\
\text { desacuerdo }\end{array}$ & 0 & 0,0 \\
$\begin{array}{l}\text { Parcialmente de } \\
\text { acuerdo }\end{array}$ & 5 & 10,0 \\
$\begin{array}{l}\text { Parcialmente de } \\
\text { acuerdo }\end{array}$ & 10 & 20,0 \\
$\begin{array}{l}\text { Altamente en } \\
\text { desacuerdo }\end{array}$ & 35 & 70,0 \\
Total & 50 & 100,0 \\
\hline
\end{tabular}

Fuente: Datos de la propia investigación

Para realizar la prueba de hipótesis se ha cruzado la información de las tablas anteriores mediante la prueba estadística Chi-cuadrado. Es decir que a través del programa SPSS, los resultados del resumen de las dimensiones del clima laboral se cruzaron, mediante la prueba estadística mencionada, con los resultados del resumen de las dimensiones de la satisfacción del cliente, las cuales se muestran en la tabla 1 y tabla 2 respectivamente.

A continuación, en la tabla 3 y tabla 4 muestran el resultado del mencionado cruce. De acuerdo con la tabla 4 , el valor de chi-cuadrado es de 12,452 con 3 grados de libertad y un valor de significancia de 0,006; como este valor es inferior a 0,05 existen razones suficientes para afirmar que los resultados en estos dos grupos de estudio no son similares, es decir, las diferencias que se observan en los porcentajes de ambos grupos son significativas, lo cual se puede observar en la tabla 3 . En consecuencia, el clima laboral en Daruchi Solutions S.A.C. no tiene relación con la satisfacción del cliente externo de Daruchi Solutions S.A.C.

Es necesario enfatizar que la hipótesis de la presente investigación no se cumple, debido a que el tamaño de la muestra fue uno de los factores determinantes para dicho resultado. Es decir, el tamaño de la muestra fue limitante, ya que al ser Daruchi Solutions S.A.C. una MYPE, solo se pudo utilizar su población como el tamaño de la muestra, en este caso en particular, por lo tanto, cabe resaltar que la muestra no fue aleatoria, sino fue seleccionada exclusivamente a conveniencia de la presente investigación. Este tipo de muestra es la no probabilística, tal como mencionan Hernández, Fernández y Baptista (2014), estas son utilizadas para "determinados diseños de estudio que requieren no tanto una "representatividad" de elementos de una población, sino una cuidadosa y controlada elección de casos con ciertas características especificadas previamente en el planteamiento del problema." 
Tabla 3

Variable: Clima Laboral * Variable: Satisfacción del Cliente

\begin{tabular}{|c|c|c|c|c|c|c|}
\hline & & \multicolumn{5}{|l|}{ Respuestas } \\
\hline & & $\begin{array}{l}\text { Altamente en } \\
\text { desacuerdo }\end{array}$ & $\begin{array}{l}\text { Parcialmente } \\
\text { en desacuer- } \\
\text { do }\end{array}$ & $\begin{array}{l}\text { Parcialmente } \\
\text { de acuerdo }\end{array}$ & $\begin{array}{l}\text { Altamente } \\
\text { de acuerdo }\end{array}$ & Total \\
\hline \multirow[t]{2}{*}{ Clima laboral } & Recuento & 1 & 7 & 44 & 38 & 90 \\
\hline & & $1,1 \%$ & $7,8 \%$ & $48,9 \%$ & $42,2 \%$ & $100,0 \%$ \\
\hline \multirow{2}{*}{$\begin{array}{l}\text { Satisfacción } \\
\text { del cliente }\end{array}$} & Recuento & 0 & 5 & 10 & 35 & 50 \\
\hline & & $0,0 \%$ & $10,0 \%$ & $20,0 \%$ & $70,0 \%$ & 100,0 \\
\hline \multirow[t]{2}{*}{ Total } & Recuento & 1 & 12 & 54 & 73 & 140 \\
\hline & & $0,7 \%$ & $8,6 \%$ & $38,6 \%$ & $52,1 \%$ & $100,0 \%$ \\
\hline
\end{tabular}

Fuente: Datos de la propia investigación.

Tabla 4

Pruebas de chi-cuadrado

\begin{tabular}{llll}
\hline & Valor & Df & $\begin{array}{l}\text { Significación } \\
\text { asintótica (bilateral) }\end{array}$ \\
\hline $\begin{array}{l}\text { Chi-cuadrado } \\
\text { de Pearson }\end{array}$ & $12,452 a$ & 3 & 0,006 \\
$\begin{array}{l}\text { Razón de } \\
\text { verosimilitud }\end{array}$ & 13,365 & 3 & 0,004 \\
$\begin{array}{l}\text { Asociación } \\
\text { lineal por lineal }\end{array}$ & 5,375 & 1 &, 020 \\
$\begin{array}{l}\text { N de casos } \\
\text { válidos }\end{array}$ & 140 & & \\
\hline
\end{tabular}

Fuente: Datos de la propia investigación.
La Tabla 5 muestra el resumen de los resultados al realizar las pruebas de chi-cuadrado para hallar la relación entre las dimensiones del clima laboral con la variable satisfacción del cliente. Se observan valores arrojados por el programa SPSS, los cuales permitieron determinar la existencia o falta de una relación entre las dimensiones del clima laboral y la variable satisfacción del cliente. Para determinar dicha relación se comparó la significación asintótica (bilateral) con el valor $\mathrm{p}$. Para aquellas dimensiones cuyo valor de significación asintótica fue inferior al valor $p(0,05)$, se pudo afirmar que los resultados en los dos grupos de estudio no son similares, por lo tanto, no hay relación; tal es el caso de las dimensiones: responsabilidad, recompensa, riesgo, estándares de desempeño y conflicto. Por el contrario, para aquellas dimensiones cuyo valor de la

Tabla 5

Pruebas de chi-cuadrado dimensiones del Clima laboral con variable Satisfacción del cliente

\begin{tabular}{llllll}
\hline $\begin{array}{l}\text { Dimensión de } \\
\text { la variable clima } \\
\text { laboral }\end{array}$ & Valor & df & $\begin{array}{l}\text { Chi Cuadrado de Pearson } \\
\text { Significación } \\
\text { asintótica (bila- } \\
\text { teral) }\end{array}$ & $\begin{array}{l}\text { Valor } p \\
(0,05) \text { Vs. } \\
\text { Signif. asintó- }\end{array}$ & Interpretación \\
\hline tica & & \\
\hline Estructura & $4,154 a$ & 2 & 0,125 & $0,125>0,05$ & Sí hay relación \\
Responsabilidad & $7,105 a$ & 2 &, 029 & $0,029<0,05$ & No hay relación \\
Recompensa & $9,990 a$ & 3 &, 019 & $0,019<0,05$ & No hay relación \\
Riesgo & $7,105 a$ & 2 &, 029 & $0,029<0,05$ & No hay relación \\
Calor & $1,385 a$ & 2 &, 500 & $0,500>0,05$ & Sí hay relación \\
Apoyo & $2,551 a$ & 2 &, 279 & $0,279>0,05$ & Sí hay relación \\
$\begin{array}{l}\text { Estándares de } \\
\text { desempeño }\end{array}$ & $10,731 a$ & 2 &, 005 & & No hay relación \\
Conflicto & $10,458 a$ & 2 &, 005 & $0,005<0,05$ & No hay relación \\
Identidad & $2,551 a$ & 2 &, 279 & $0,279>0,05$ & Sí hay relación \\
\hline
\end{tabular}


significación asintótica (bilateral) fue superior al valor $p$ $(0,05)$, se pudo para afirmar que los resultados en los dos grupos de estudio son similares, por lo tanto, hay relación; tal es el caso de las dimensiones: estructura, calor, apoyo e identidad.

\section{DISCUSIÓN}

De acuerdo al objetivo de determinar la relación entre el clima laboral con la satisfacción del cliente externo de Daruchi Solutions S.A.C. como parte de su estrategia de internacionalización, se observó en la prueba de hipótesis que el valor chi-cuadrado es de 12,452 con 3 grados de libertad y un valor de significancia de 0,006 , como los valores de significancia son inferiores a 0,05 podemos afirmar que existen razones suficientes para aseverar que los resultados en estos dos grupos de estudio no son similares, es decir, que las diferencias que se observan en los porcentajes de ambos grupos son significativas, por lo tanto, el clima laboral en Daruchi Solutions S.A.C. no tiene relación con la satisfacción del cliente externo de Daruchi Solutions S.A.C. En otras palabras, no se cumple la hipótesis de la presente investigación. De manera contraria, en la investigación de Novoa y Regalado (2017) se halla un resultado diferente posiblemente debido a la diferencia en el tamaño de las muestras. En este sentido, se concluye que dentro de la empresa Oltursa, existe una relación entre el clima laboral y la satisfacción del cliente, es decir, a mayor percepción positiva del clima laboral, mayor será la satisfacción del cliente, y viceversa. Además, el $81,9 \%$ de sus trabajadores tiene una percepción entre favorable y muy favorable, en donde destaca el compromiso de los trabajadores para cumplir con las metas establecidas, lo que permitirá brindar un mejor servicio al cliente.

Asimismo, según los resultados del trabajo de investigación de Echezuría y Rivas (2001), se encontró que la dimensión estructura dentro de los organismos públicos, en este caso alcaldías, está relativamente bien definida, puesto que se obtuvo un $69 \%$ de favorabilidad; es decir, tres alcaldías de cinco realmente se preocupan de que sus trabajadores tengan claro cuáles son sus funciones, así como cuáles son las normas, políticas y procedimientos de dicho organismo. Sucede todo lo contrario con respecto a las empresas privadas, con una favorabilidad del $98 \%$, revela que estas en estas organizaciones predominan el cumplimiento de las normas y el seguimiento de los canales regulares en la realización de las actividades, además de que los trabajadores conocen a la perfección la estructura organizativa y las políticas de la empresa. Por lo tanto, se infiere que en el sector público tienden a enfatizar en los trámites, teniendo exceso de reglas, y que muchas veces este es un factor que no permite que las tareas sean realizadas eficientemente. Mientras que en el sector privado sí se tiene claro los canales. De manera que para nuestra investigación, la percepción de la estructura dentro de la empresa tiene una relación di- recta con la satisfacción del cliente, de la misma manera que para las empresas privadas, según los resultados de la investigación de Echezuría y Rivas (2001), se puede inferir que la estructura tiene una influencia en la satisfacción del cliente, ya que al tener claras cuáles son las normas funciones y políticas de la empresa, los trabajadores son capaces de realizar sus tareas de manera eficiente, en consecuencia, los clientes reciben un mejor servicio, logrando así su satisfacción.

Por otro lado, en la presente investigación se infiere que, al no existir una similitud entre los resultados de la apreciación de la recompensa y la satisfacción del cliente, estos no tienen una relación directa. Mientras que en la investigación de Sierra (2015) se muestra que 12 colaboradores de 29 "consideran que su trabajo no está bien remunerado y que no se encuentra en consonancia con los sueldos de su entorno social, ni con la labor que realizan" (p.55). Por lo que la autora recomienda al Hospital Regional de Cobán, A.V. debe proponer una valuación de puestos, para que la remuneración sea acorde a las funciones y puestos que ocupan los colaboradores. Los resultados señalados anteriormente pertenecen al factor remuneración, el cual se puede considerar como equivalente a la dimensión recompensa. Por lo tanto, se puede inferir, que al tratarse de una entidad que ofrece servicios de salud, la percepción de cómo el trabajo de los colaboradores es recompensado influye en cómo estos realizan su trabajo, el cual afecta directamente al cliente o paciente en este caso.

Para Pelaes (2010) existe una relación directa entre el clima organizacional y la satisfacción del cliente en la empresa Telefónica del Perú. Esta conclusión se da después de observar que se encontró una correlación de 0.37 entre la dimensión de claridad y coherencia de la dirección y la satisfacción del cliente. Un resultado diferente se encontró en la presente investigación, por lo tanto, la claridad que tienen los trabajadores sobre las normas de trabajo estipuladas por la dirección no tiene relación con lograr la satisfacción del cliente.

Conclusiones: No hay relación entre la variable clima laboral y la variable satisfacción del cliente; al margen de ello se puede concluir que el clima laboral de Daruchi Solutions es favorable ya que el porcentaje de los trabajadores que están entre parcialmente y altamente de acuerdo con el clima laboral es del 91\% (tabla 2), eso significa que la empresa cumple con una de las dimensiones necesarias para la internacionalización. Es necesario que la empresa mantenga ese nivel y realice mejoras en aquellas dimensiones deficientes, mencionadas anteriormente. Además de considerar el clima laboral, es importante también tener en cuenta otras dimensiones determinantes para la internacionalización, tales como la administrativa, la financiera, la económica, etc. Una vez que la empresa las analice y corrobore su nivel idóneo, estará en el momento adecuado para seguir adelante con los procesos para ser 
una importadora, como parte de su intención de internacionalización.

\section{REFERENCIAS}

Cortés, N. (2009). Diagnóstico del clima organizacional. Hospital "Dr. Luis F. Nachón”. Xalapa, Ver., 2009 (Tesis para optar el grado de magíster en Salud Pública). Universidad Veracruzana, Veracruz, México.

Echegaray, V. (2014). Relación entre el clima organizacional y la satisfacción del cliente de la CMAC Tacna en el año 2013 (Tesis para optar el grado de maestro en ciencias con mención en Gestión Empresarial). Universidad Nacional Jorge Basadre Grohmann - Tacna, Perú.

Echezuría, A., \& Rivas, A. (2001). Estudio de clima organizacional en la unidad de recursos humanos en una muestra de organismos públicos y empresas privadas (Tesis para optar al título de licenciado en Relaciones Industriales). Universidad Católica Andrés Bello, Caracas, Venezuela.

Hernández, R., Fernández, C., \& Baptista, P. (2014). Selección de la muestra. En Metodología de la Investigación (6 $6^{a}$ ed.). México: McGraw-Hill. Las páginas no son necesarias, es ciudad no país.

Matsumoto, R. (2014). Desarrollo del Modelo Servqual para la medición de la calidad de servicio en la empresa de publicidad Ayuda Experto. Perspectivas, 17 (33), 181- 209.

Montoya, D. (2016). Relación entre el clima organizacional y la evaluación del desempeño personal en una empresa de servicios turísticos: casos PTS Perú 2015 (Tesis para optar el grado de magíster en Relaciones Laborales). Pontificia Universidad Católica del Perú, Lima, Perú.

Novoa, M., \& Regalado, T. (2017). Análisis de la relación entre clima laboral y satisfacción del cliente en la empresa Oltursa - Piura y Chaclacayo (Tesis para optar el título de licenciado en Administración de Empresas). Universidad de Piura, Perú.

Parasuraman, A., Zeithaml, V. A., \& Berry, L. L. (1988). Servqual: A multiple-item scale for measuring consumer perceptions of service quality. Journal of retailing, 64(1), 12-40.

Pelaes, O. (2010). Relación entre el clima organizacional y la satisfacción del cliente en una empresa de servicios telefónicos (Tesis para optar el grado de doctor en Ciencias Administrativas). Universidad Nacional Mayor de San Marcos, Perú.

Sierra, M. (2015). El clima laboral en los/as colaboradores/as del área administrativa del hospital regional de Cobán, A.V (Tesis para optar el título de licenciado en Psicología Industrial / Organizacional). Universidad Rafael Landívar, Alta Verapaz, Guatemala.
Correo electrónico para correspondencia:

christinagh95@gmail.com 